\title{
Rotation Modes Stability Analysis and Phase Compensation for Magnetically Suspended Flywheel Systems with Cross Feedback Controller and Time Delay
}

\author{
Yuan Ren, ${ }^{1}$ Xiaocen Chen, ${ }^{1,2}$ Yuanwen Cai, ${ }^{1}$ and Weijie Wang ${ }^{1}$ \\ ${ }^{1}$ Equipment Academy, Beijing 101416, China \\ ${ }^{2}$ Beijing Institute of Special Electromechanical Technology, Beijing 100012, China \\ Correspondence should be addressed to Yuan Ren; renyuan_823@aliyun.com
}

Received 12 December 2015; Accepted 8 February 2016

Academic Editor: Yuri Vladimirovich Mikhlin

Copyright (C) 2016 Yuan Ren et al. This is an open access article distributed under the Creative Commons Attribution License, which permits unrestricted use, distribution, and reproduction in any medium, provided the original work is properly cited.

\begin{abstract}
This paper analyzes the effects of time delay on the stability of the rotation modes for the magnetically suspended flywheel (MSFW) with strong gyroscopic effects. A multi-input multioutput system is converted into a single-input single-output control system with complex coefficient by variable reconstruction, and the stability equivalence of the systems before and after variable reconstruction is proven. For the rotation modes, the stability limits and corresponding vibration frequencies are found as a function of nondimensional magnetic stiffness and damping and nondimensional parameters of rotor speed and time delay. Additionally, the relationship between cross feedback control system stability and time delay is investigated. And an effective phase compensation method based on cross-channel is further presented. Simulation and experimental results are presented to demonstrate the correctness of the stability analysis method and the superiority of the phase compensation strategy.
\end{abstract}

\section{Introduction}

With the development of magnetic bearing technology, a magnetically suspended flywheel (MSFW) represents an exciting alternative to the traditional mechanical flywheel due to its inherent superior features such as contact free operation, zero friction, adjustable damping, and stiffness as well as the potential for low vibration and super high rotational speeds $[1,2]$. As a result, it has been widely employed in power industry [3,4], transportation, spaceflight $[5-8]$, and so on $[9,10]$.

To maximize the energy storage capacity, a MSFW usually chooses a radially thick rotor so that the principle mass moment of inertial is larger than the transversal counterpart, that is, $J_{z} / J_{x}>1$. In this case, the gyroscopic effects are especially strong, which puts a strain on the stability analysis of the system.

Particularly, to improve the transient response and to reduce power consumption of a magnetic bearing, the permanent-magnetic-biased hybrid magnetic bearings
(PMHMBs) are applied [1]. This bias field causes the rotor to behave as it was supported on a spring with negative stiffness. There is no time delay associated with the bias field since it is caused by a permanent magnet. However, the time delay of the electromagnetic bearing always exists since each of the components involved in it and its control system have a time delay associated with them. For an analog device, the time delay can be approximated by a linear time delay low-pass filter. The amplitude of the transfer function is assumed to be a constant and the phase is linear. However, for the digital devices, this time delay is the loop time for each measurement [11]. The total time delay is the sum of the individual time delay [12]. All of these make the stability analysis of the MSFW more complex and difficult.

In recent years, considerable researches have been carried out for the stability analysis of the rotor supported by magnetic bearings with time delay. Ji $[13,14]$ analyzed the effect of time delays on the nonlinear dynamic behavior of the magnetic bearing system. Wang and Liu [15], Li et al. [16], and Ji [17] studied the Hopf bifurcation and its stability in 


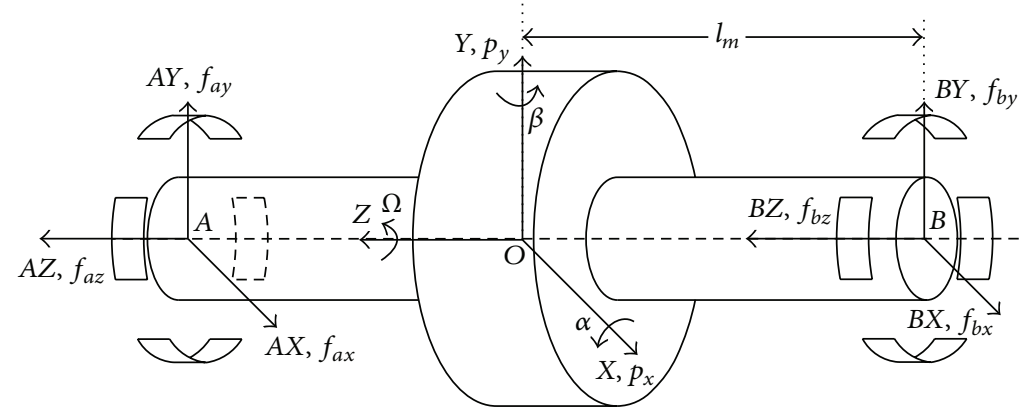

FIGURE 1: Force and coordinate system definition of a MSFW.

a magnetic bearing system with time delays. However, these works only considered a simple single-degree-of-freedom (DOF) magnetic bearing without gyroscopic effects. As for the multi-DOF cases with high rotor speed and strong gyroscopic effects, Kascak et al. [18] employed nondimensional magnetic stiffness and damping as well as nondimensional system parameters to describe the stability limits of a PD controller. However, they did not consider the cross feedback controller. Kascak et al. [19] presented the stability boundaries of a spinning rotor with parametrically excited gyroscopic system. Bauomy [20] performed the stability analysis of a rotor active magnetic bearing system with time varying stiffness. Inayat-Hussain [21] studied the nonlinear dynamics of a magnetically supported rigid rotor in auxiliary bearings. Andersen et al. [22] and Sugai et al. [23] analyzed the rotation modes stability of the magnetically suspended rotor with passive cylinder-magnet bearings. Additionally, Pei [24] presented the stability boundaries of a spinning rotor with parametrically excited gyroscopic system. Fan and Pan [25] described the stability of rotor-shaft system including electromagnetic exciters. All in all, few literatures studied the multi-DOF magnetic bearings with time delays and gyroscopic effects.

To resolve the gyroscopic effects, considerable research has been conducted and various approaches have been proposed [26-32]. Among these methods, decentralized PD plus filtered cross feedback control is the most well-known and easy-to-implement approach [29]. To further improve the system nutation stability, different phase compensation methods are developed, such as unsymmetrical current sampling resistance method $[11,12]$ and inverse system method with phase compensation $[33,34]$, where the phase compensations are performed with both decentralized and cross-channels. For simplicity, it is called the combinational compensation method in this paper. In fact, there are other phase compensation strategies in addition to this, such as phase compensation with decentralized channels and phase compensation with cross-channels. Thus, the question naturally raised is what is the most effective way for the system stability among the three phase compensation methods? This has not been effectively resolved.

To resolve the problems above, this paper describes a modeling method which is used to theoretically map the stability boundaries of the MSFW controller with cross feedback and time delay. As for the rotation modes, the relationships between the time delays and the critical nutation frequency are further analytically analyzed, and then an effective phase compensation strategy is proposed.

This paper is organized as follows. First, in Section 2, the system is modeled with complex coefficient. Then, its rotation modes stability is further studied in Section 3. Simulation and experiments are developed in Section 4. Finally, Section 5 concludes this paper.

\section{Modeling of the MSFW System and Its Variable Reconstruction}

2.1. Modeling of the MSFW System. Here, we review the model of a rigid rotor supported by magnetic bearings, which has been established in [7]. Figure 1 shows the coordinate system definition of a MSFW, where $O$ is the geometric center of the magnetic bearing stator; $l_{m}$ refers to the distance between the central point of radial magnetic bearing and point $O ; \Omega$ is the rotor speed, $X-, Y$-, and $Z$-axis form the generalized coordinate system of the rotor position; $p_{x}$ and $p_{y}$ are the torques in the $X$ and $Y$ directions, respectively; $\alpha$ and $\beta$ are the rotor angular displacements about the $X$ - and $Y$-axis. $f_{a x}, f_{a y}, f_{b x}, f_{b y}, f_{a z}$, and $f_{b z}$ are the magnetic forces along the magnetic bearing coordinate system $A X-, A Y-, B X-$ , $B Y-, A Z$-, and $B Z$-axis.

Then, the dynamic model of a rigid rotor supported by PMHMBs can be described as

$$
\begin{aligned}
m \ddot{x} & =f_{a x}+f_{b x}, \\
J_{y} \ddot{\beta}-J_{z} \Omega \dot{\alpha} & =p_{y}=l_{m}\left(f_{a x}-f_{b x}\right), \\
m \ddot{y} & =f_{a y}+f_{b y}, \\
J_{x} \ddot{\alpha}+J_{z} \Omega \dot{\beta} & =p_{x}=l_{m}\left(f_{b y}-f_{a y}\right), \\
m \ddot{z} & =f_{a z}+f_{b z},
\end{aligned}
$$

where $m$ is the mass of the flywheel rotor; $J_{x}, J_{y}$, and $J_{z}$ are the moments of inertia of the rotor about the $X$-, $Y$-, and $Z$-axis, respectively, and $J_{x}=J_{y}=J_{r}$; and $x, y$, and $z$ are the linear displacements of the mass center of the rotor from point $O$ in the $X-, Y$-, and $Z$-axis, respectively.

Figure 2 shows the schematic of the cross feedback control system, where $g_{b}(s), g_{\mathrm{cr}}(s)$, and $g_{f}(s)$ are the transfer 


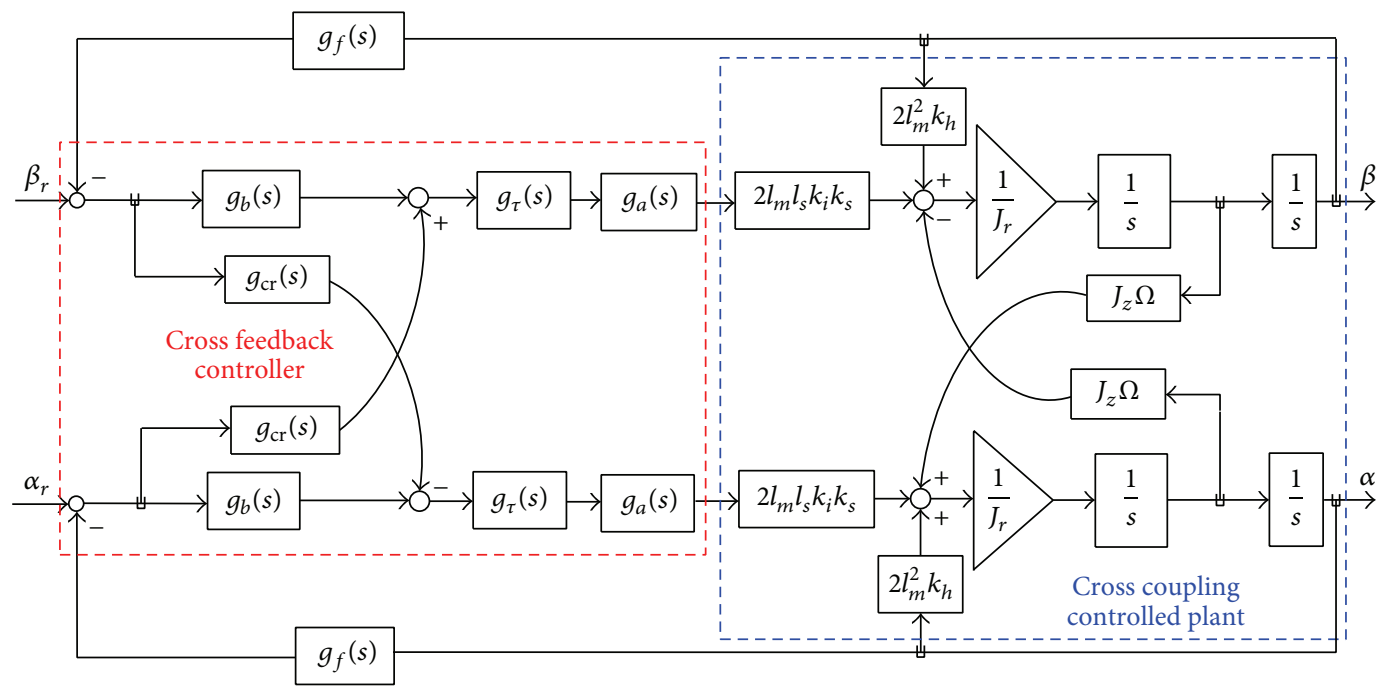

FIGURE 2: Schematic diagram of the decentralized PD plus filter cross feedback controller.

functions of the basic controller (such as a PID controller), the cross feedback controller, and the antialias filter, respectively.

As for an axisymmetric rotor supported by PMHMBs, the magnetic force $f_{\lambda}$ can be linearized as [7]

$$
f_{\lambda}=k_{i \lambda} i_{\lambda}+k_{h \lambda} h_{\lambda}, \quad \lambda=a x, a y, b x, b y, a z, b z,
$$

where $k_{i \lambda}$ and $k_{h \lambda}$ denote, respectively, the current stiffness and displacement stiffness of the $\lambda$ channel. Note that the four radial channels of the MSFW are often symmetric; for simplicity, $k_{i}$ and $k_{h}$ denote the current and displacement stiffness of the radial magnetic bearings, respectively.

For PMHMBs, there is no time delay associated with the bias field since it is produced by a permanent magnet [24], and the delay only exists in the electromagnetic field. Accordingly, the closed-loop model of the control system with the cross feedback controller can be given by $[33,35]$

$$
\begin{aligned}
& m \ddot{x}(t)-2 k_{h} x(t)=-2 k_{i} k_{s} g_{b} x(t-\tau), \\
& J_{y} \ddot{\beta}(t)-J_{z} \Omega \dot{\alpha}(t)-2 k_{h} l_{m}^{2} \beta(t) \\
& \quad=-2 l_{m} l_{s} k_{i} k_{s}\left[g_{b}(\beta(t-\tau))+g_{\mathrm{cr}}(\alpha(t-\tau))\right], \\
& m \ddot{y}(t)-2 k_{h} y(t)=-2 k_{i} k_{s} g_{b} y(t-\tau), \\
& J_{x} \ddot{\alpha}(t)+J_{z} \Omega \dot{\beta}(t)-2 k_{h} l_{m}^{2} \alpha(t) \\
& \quad=-2 l_{m} l_{s} k_{i} k_{s}\left[g_{b}(\alpha(t-\tau))-g_{\mathrm{cr}}(\beta(t-\tau))\right], \\
& m \ddot{z}(t)-2 k_{h z} z(t)=-2 k_{i z} k_{s} g_{b z} z(t-\tau),
\end{aligned}
$$

where $g_{a}$ denotes the power amplifier of the HMBs, $k_{s}$ is the proportional gain of the displacement sensor, $l_{s}$ is the distance from the geometric center of the rotor to the sensor, and $\tau$ is the control system delay.

It can be seen from (3) that the cross feedback control only influences the system rotation modes stability and does not endanger the system translation modes stability. As for the translation modes of the MSFW, their stability analysis can be equivalent to that of the single-DOF magnetic bearing control system, which has been clearly analyzed. Accordingly, this paper focuses on the rotation modes stability analysis of the MSFW system with cross feedback control system.

The rotation modes model can be described as

$$
\begin{aligned}
& J_{y} \ddot{\beta}(t)-J_{z} \Omega \dot{\alpha}(t)-2 k_{h} l_{m}^{2} \beta(t) \\
& \quad=-2 l_{m} l_{s} k_{i} k_{s}\left[g_{b}(\beta(t-\tau))+g_{\mathrm{cr}}(\alpha(t-\tau))\right], \\
& J_{x} \ddot{\alpha}(t)+J_{z} \Omega \dot{\beta}(t)-2 k_{h} l_{m}^{2} \alpha(t) \\
& \quad=-2 l_{m} l_{s} k_{i} k_{s}\left[g_{b}(\alpha(t-\tau))-g_{\mathrm{cr}}(\beta(t-\tau))\right] .
\end{aligned}
$$

2.2. Variable Reconstruction and Its Stability Equivalence Analysis. As for the rotation modes model (4), it is a twoinput and two-output control system. To simplify the stability analysis of the MSFW rotation modes, variable reconstruction is employed in this paper.

From the coordinate definition of the MSFW shown in Figure 1, $\alpha$ leads $\beta 90$ degrees. Accordingly, we can define $\varphi=\alpha+j \beta$, where $j$ is the imaginary number unit and $j^{2}=-1$. Note that the dissymmetry of (4) and multiplying the second equation by $j$ and then adding the result to the fourth equation yield the differential equations:

$$
\begin{gathered}
J_{r} \ddot{\varphi}(t)-j H \dot{\varphi}(t)-2 k_{h} l_{m}^{2} \varphi(t)+P \varphi(t-\tau) \\
+D \dot{\varphi}(t-\tau)+j k_{c} H \dot{\varphi}(t-\tau)=0,
\end{gathered}
$$

where $P=2 l_{m} l_{s} k_{i} k_{s} k_{p}, D=2 l_{m} l_{s} k_{i} k_{s} k_{d}$.

According to the control system theory, the necessary and sufficient conditions of the rotation modes stability are as follows:

$$
\begin{aligned}
& \lim _{t \rightarrow \infty} \alpha(t)=0, \\
& \lim _{t \rightarrow \infty} \beta(t)=0 .
\end{aligned}
$$


Accordingly,

$$
\lim _{t \rightarrow \infty}(\alpha(t)+j \beta(t))=0 .
$$

As for complex-coefficient system (5), the necessary and sufficient condition of the stable control system is

$$
\lim _{t \rightarrow \infty} \phi(t)=0 .
$$

Note that $\phi(t)=\alpha(t)+j \beta(t)$; that is, $\lim _{t \rightarrow \infty} \alpha(t)=0$ and $\lim _{t \rightarrow \infty} \beta(t)=0$.

Therefore, the stability of system (4) is equivalent to that of (5). Then, a two-input two-output system is converted into a single-input single-output control system with complex coefficient. That is, we can get the stability of system (4) by analyzing the stability of system (5).

\section{Stability Analysis of Rotation Modes of the MSFW}

3.1. Stability Analysis of the Rotation Modes. According to the classical small signal stability analysis method, assuming an eigenvalue solution of the form $\varphi(t)=A e^{\lambda t}$, then

$$
\varphi(t-\tau)=A e^{\lambda(t-\tau)} .
$$

Substituting (9) into (5) yields the characteristic equation:

$$
\begin{aligned}
& J_{r} \lambda^{2}-j H \lambda-2 k_{h} l_{m}^{2}+P e^{-\lambda \tau}+D \lambda e^{-\lambda \tau}+j k_{c} H \lambda e^{-\lambda \tau} \\
& \quad=0 .
\end{aligned}
$$

Define the nondimensional parameters as follows:

$$
\begin{aligned}
\mu & =\lambda \tau, \\
\tau_{0}^{2} & =\frac{P \tau^{2}}{J_{r}}, \\
2 \xi_{1} \tau_{0} & =\frac{D \tau}{J_{r}}, \\
2 \xi_{2} \tau_{0} & =\frac{k_{c} H \tau}{J_{r}}, \\
\tau_{n}^{2} & =\frac{2 k_{h} l_{m}^{2} \tau^{2}}{J_{r}}, \\
\tau \eta & =\frac{\tau H}{J_{r}} .
\end{aligned}
$$

Then, the eigenvalue equation can be simplified as follows:

$$
\left(\mu^{2}-j \tau \eta \mu-\tau_{n}^{2}\right) e^{u}+2 \tau_{0}\left(\xi_{1}+j \xi_{2}\right) \mu+\tau_{0}^{2}=0 .
$$

The eigenvalue equation has the similar solution to that of the translation mode under the condition the rotor speed of the MSFW is zero. If the eigenvalue is complex, then the imaginary part of the eigenvalue is the circular frequency of the vibration that multiplies the time delay; that is

$$
\mu=\alpha+j \beta=\alpha+j \omega \tau
$$

Substituting (13) into (12) yields

$$
\begin{array}{r}
\operatorname{Im}\left(\left(\mu^{2}-j \tau \eta \mu-\tau_{n}^{2}\right) e^{u}\right)+2 \tau_{0} \xi_{1} \beta+2 \tau_{0} \xi_{2} \alpha=0, \\
\operatorname{Re}\left(\left(\mu^{2}-j \tau \eta \mu-\tau_{n}^{2}\right) e^{u}\right)+2 \tau_{0} \xi_{1} \alpha-2 \tau_{0} \xi_{2} \beta+\tau_{0}^{2}=0 .
\end{array}
$$

Solving the real and imaginary portions of the eigenvalue equation, the nondimension damping obtains

$$
2 \tau_{0} \xi_{1}=\frac{-\operatorname{Im}\left(\left(\mu^{2}-j \tau \eta \mu-\tau_{n}^{2}\right) e^{u}\right)}{\beta}-\frac{2 \tau_{0} \xi_{2} \alpha}{\beta} .
$$

And the nondimensional stiffness achieves

$$
\tau_{0}^{2}=-\operatorname{Re}\left(\left(\mu^{2}-j \tau \eta \mu-\tau_{n}^{2}\right) e^{u}\right)+2 \tau_{0} \varepsilon_{2} \beta .
$$

If the real part of the eigenvalue $\alpha$ is positive, the vibrations grow in time and the system is unstable. Contrarily, if $\alpha$ is negative, the vibrations decay in time and the system is stable. The case that $\alpha$ equals zero defines the stability boundary as follows:

$$
\begin{aligned}
2 \tau_{0} \xi_{1} & =\frac{\left(\beta^{2}-\tau \eta \beta+\tau_{n}^{2}\right) \sin \beta}{\beta}, \\
\tau_{0}^{2} & =\left(\beta^{2}-\tau \eta \beta+\tau_{n}^{2}\right)\left(\cos \beta+\frac{\xi_{2}}{\xi_{1}} \sin \beta\right) .
\end{aligned}
$$

Note that this paper mainly focuses on the nutation modes stability analysis and phase compensation for MSFW with cross feedback controller and time delay, the possible hidden oscillations [36], Bifurcation and global stability analysis being the next topics in the near future.

3.2. Relationship between Cross Feedback Control System Stability and Time Delay. To further analyze how the control system delay influences the nutation stability, the following analysis has been developed, and an effective phase compensation strategy is proposed.

The eigenvalue equation of (5) can be resolved as

$$
\begin{aligned}
& J_{r} \lambda^{2}-j H \lambda-2 k_{h} l_{m}^{2}+P e^{-\lambda \tau}+D \lambda e^{-\lambda \tau}+j H \lambda e^{-\lambda \tau} \\
& \quad=0 .
\end{aligned}
$$

Suppose its eigenvalue is $\lambda=\sigma+\mathrm{j} \omega$; then, substituting this into (18) yields

$$
\begin{gathered}
J_{r}\left(\sigma^{2}-\omega^{2}\right)+H \omega-2 k_{h} l_{m}^{2}+P e^{-\sigma \tau_{1}} \cos \left(\omega \tau_{1}\right) \\
+D \sigma e^{-\sigma \tau_{1}} \cos \left(\omega \tau_{1}\right)+M \omega e^{-\sigma \tau_{1}} \sin \left(\omega \tau_{1}\right) \\
-H \omega \cos \left(\omega \tau_{2}\right)+H \sigma \sin \left(\omega \tau_{2}\right)=0, \\
2 J_{r} \sigma \omega-H \sigma-P e^{-\sigma \tau_{1}} \sin \left(\omega \tau_{1}\right) \\
+D e^{-\sigma \tau_{1}}\left(\omega \cos \left(\omega \tau_{1}\right)-\sigma \sin \left(\omega \tau_{1}\right)\right) \\
+H \sigma \cos \left(\omega \tau_{2}\right)+H \omega \sin \left(\omega \tau_{2}\right)=0 .
\end{gathered}
$$


(1) If $\sigma=\omega=0$, then we have $L=2 k_{h} l_{m}^{2}$. The system has a simple steady state bifurcation.

(2) If $\sigma=0, \omega \neq 0$, the system is critically stable; (19) can be simplified as

$$
\begin{aligned}
& P \cos \left(\omega \tau_{1}\right)+D \omega \sin \left(\omega \tau_{1}\right)-H \omega \cos \left(\omega \tau_{2}\right) \\
& =J_{r} \omega^{2}-H \omega+2 k_{h} l_{m}^{2}
\end{aligned}
$$

$-P \sin \left(\omega \tau_{1}\right)+D \omega \cos \left(\omega \tau_{1}\right)+H \omega \sin \left(\omega \tau_{2}\right)=0$.

Moving the terms of trigonometric functions into the lefthand side and then squaring and adding these two equations yield a quadratic equation for $\omega$ :

$$
\begin{aligned}
P^{2}+ & D^{2} \omega^{2}+H^{2} \omega^{2}-2 P H \omega \cos \left(\tau_{1}-\tau_{2}\right) \\
& -2 D H \omega^{2} \sin \left(\omega \tau_{1}-\omega \tau_{2}\right) \\
= & \left(J_{r} \omega^{2}-H \omega+2 k_{h} l_{m}^{2}\right)^{2} .
\end{aligned}
$$

When $\tau_{1}=\tau_{2}$, the above equation can be further simplified as

$$
\begin{aligned}
P^{2} & +D^{2} \omega^{2}+H^{2} \omega^{2}-2 P H \omega \\
& =\left(J_{r} \omega^{2}-H \omega+2 k_{h} l_{m}^{2}\right)^{2} .
\end{aligned}
$$

Sumptuously, multiplying $\sin \omega \tau_{2}$ and $\cos \omega \tau_{2}$ to the two equations of (19) and then adding them together yield

$$
\begin{aligned}
& P \sin \left(\omega \tau_{1}-\omega \tau_{2}\right)-D \omega \cos \left(\omega \tau_{1}-\omega \tau_{2}\right) \\
& +\left(J_{r} \omega^{2}-H \omega+2 k_{h} l_{m}^{2}\right) \sin \left(\omega \tau_{2}\right)=0 .
\end{aligned}
$$

Note that $\tau_{1}=\tau_{2}$ as, for a MSFW control system without phase compensation, (23) can be rewritten as

$$
D \omega=\left(J_{r} \omega^{2}-H \omega+2 k_{h} l_{m}^{2}\right) \sin \left(\omega \tau_{2}\right) .
$$

Accordingly, the second equation of (20) achieves

$$
(H \omega-P) \sin (\omega \tau)+D \omega \cos (\omega \tau)=0 .
$$

Note that, for the MSFW with high rotor speed, $H \omega \gg P$. As for positive $\omega$, we have

$$
\begin{aligned}
& \cos (\omega \tau)<0, \\
& \sin (\omega \tau)>0 .
\end{aligned}
$$

That is, $\omega \tau \in(2 k \pi+\pi / 2,2 k \pi+\pi), k=0,1,2, \ldots$

Combining (24) and (26) yields

$$
J_{r} \omega^{2}-H \omega+2 k_{h} l_{m}^{2}>0 .
$$

Combing (25) and (26) obtains

$$
\tau_{2}=\frac{\pi-\arctan (D \omega /(H \omega-P))}{\omega} .
$$

Deriving the above equation with $\omega$ yields

$$
\begin{aligned}
& \frac{d \tau_{2}}{d \omega} \\
& =\frac{D P \omega /\left(d^{2} \omega^{2}+(H \omega-P)^{2}\right)+\arctan (D \omega /(H \omega-P))-\pi}{\omega^{2}} \\
& <\frac{D P \omega / 2 D \omega(H \omega-P)+\arctan (D \omega /(H \omega-P))-\pi}{\omega^{2}} .
\end{aligned}
$$

Note that $H \omega \gg P$ and $H \gg D$ in practice; therefore, $d \tau_{2} / d \omega<0$. That is, the larger the delay time is, the smaller the critical nutation frequency is.

Theorem 1. If the rotor speed is limited to zero, $\lim _{\varphi \rightarrow 0^{-}}\left|\left(\partial \varphi / \partial \tau_{2}\right) /\left(\partial \varphi / \partial \tau_{1}\right)\right| \ll 1$, and if the rotor speed is high enough, $\lim _{\varphi \rightarrow 0^{-}}\left|\left(\partial \varphi / \partial \tau_{2}\right) /\left(\partial \varphi / \partial \tau_{1}\right)\right| \gg 1$.

Proof. Multiplying the first and the second item of (25) by $\sin \left(\omega \tau_{2}\right)$ and $\cos \left(\omega \tau_{2}\right)$, respectively, and then adding them together yields

$$
\begin{aligned}
& \varphi \\
& =\arctan \frac{D \omega \cos \left(\omega \tau_{1}\right)-P \sin \left(\omega \tau_{1}\right)+H \omega \sin \left(\omega \tau_{2}\right)}{P \cos \left(\omega \tau_{1}\right)+D \omega \sin \left(\omega \tau_{1}\right)-H \omega \cos \left(\omega \tau_{2}\right)} \\
& \quad+n \pi .
\end{aligned}
$$

Then,

$$
\frac{\partial \varphi}{\partial \tau_{1}}=\frac{1}{1+\xi^{2}} \frac{\left(-D \omega^{2} \sin \left(\omega \tau_{1}\right)-P \omega \cos \left(\omega \tau_{1}\right)\right) \gamma-\left(-P \sin \left(\omega \tau_{1}\right)+D \omega^{2} \cos \left(\omega \tau_{1}\right)\right) \eta}{\gamma^{2}}
$$

where

$$
\begin{aligned}
& \gamma=P \cos \left(\omega \tau_{1}\right)+D \omega \sin \left(\omega \tau_{1}\right)-H \omega \cos \left(\omega \tau_{2}\right), \\
& \eta=D \omega \cos \left(\omega \tau_{1}\right)-P \sin \left(\omega \tau_{1}\right)+H \omega \sin \left(\omega \tau_{2}\right), \\
& \xi=\frac{D \omega \cos \left(\omega \tau_{1}\right)-P \sin \left(\omega \tau_{1}\right)+H \omega \sin \left(\omega \tau_{2}\right)}{P \cos \left(\omega \tau_{1}\right)+D \omega \sin \left(\omega \tau_{1}\right)-H \omega \cos \left(\omega \tau_{2}\right)} .
\end{aligned}
$$

Therefore,

$$
\lim _{\varphi \rightarrow 0^{-}} \frac{\eta}{\gamma}=0
$$

Hence,

$$
\lim _{\varphi \rightarrow 0^{-}} \frac{\partial \varphi / \partial \tau_{2}}{\partial \varphi / \partial \tau_{1}}=\frac{H \omega^{2} \cos (\omega \tau)}{-D \omega^{2} \sin (\omega \tau)-P \omega \cos (\omega \tau)} .
$$


When $\varphi=0$, we have

$$
D \omega \cos (\omega \tau)-P \sin (\omega \tau)+H \omega \sin (\omega \tau)=0
$$

Noting that $\cos (\omega \tau) \neq 0$ yields

$$
\tan (\omega \tau)=\frac{D \omega}{P-H \omega} .
$$

Substituting (36) into (34) yields

$$
\begin{aligned}
\lim _{\varphi \rightarrow 0^{-}} \frac{\partial \varphi / \partial \tau_{2}}{\partial \varphi / \partial \tau_{1}} & =\frac{H \omega^{2}}{-D \omega^{2} \tan (\omega \tau)-P \omega} \\
& =\frac{H \omega^{2}}{-D^{2}\left(\omega^{3} /(P-H \omega)\right)-P \omega} \\
& =\frac{H \omega(P-H \omega)}{P H-P^{2}-D^{2} \omega^{2}} .
\end{aligned}
$$

Obviously, if the rotor speed is limited to zero, $\lim _{\varphi \rightarrow 0^{-}}\left|\left(\partial \varphi / \partial \tau_{2}\right) /\left(\partial \varphi / \partial \tau_{1}\right)\right| \ll 1$, compared with the cross-channel, the decentralized channel is the main factor which endangers the system nutation stability. If the rotor speed is high enough, $\lim _{\varphi \rightarrow 0^{-}}\left|\left(\partial \varphi / \partial \tau_{2}\right) /\left(\partial \varphi / \partial \tau_{1}\right)\right| \gg 1$, the delay time of the cross-channel is the main factor which influences the system nutation stability. This completes the proof.

According to Theorem 1, we can further draw the following corollary.

Corollary 2. As for the control system (23), if $\tau_{2}=0$, then the stability of the rotation modes of the MSFW has nothing to do with the rotor speed.

Proof. Since $\tau_{2}=0,(24)$ can be simplified as

$$
J_{r} \ddot{\varphi}(t)-2 k_{h} l_{m}^{2} \varphi(t)+P \varphi\left(t-\tau_{1}\right)+D \dot{\varphi}\left(t-\tau_{1}\right)=0 .
$$

The stability analysis is similar to that of the translation modes of the MSFW. This completes the proof.

Accordingly, as for the MSFW system with high rotor speed, it is the delay time of the cross-channel that influences seriously the system nutation stability instead of the decentralized channel. As a result, cross-channel phase compensation is more effective than that of the decentralized channel at high speeds and the nutation stability of the MSFW can be further improved.

Similarly, it can be proven that the cross-channel compensation method is more effective than that of the combinational compensation method. Accordingly, at high rotor speeds, cross-channel compensation is the most effective phase compensation method of the three compensation methods.

\section{Simulation and Experimental Study}

In order to demonstrate the effectiveness and validity of the proposed stability analysis and phase compensation strategy, and to reveal how closely the theory represents the physical system, both simulation and experiments have been developed.

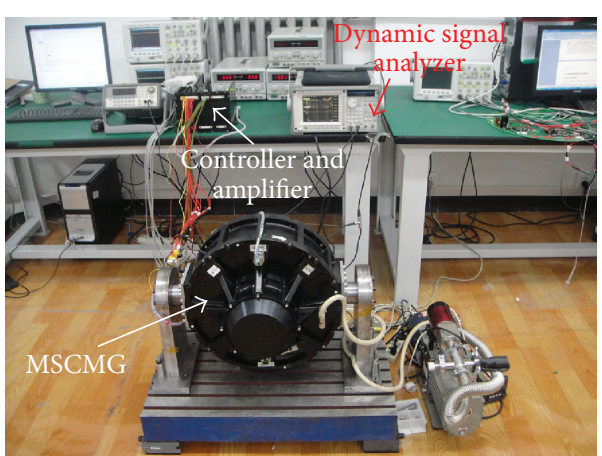

FIGURE 3: Photograph of the fabricated laboratory setup.

TABLE 1: System parameters of the MSCMG.

\begin{tabular}{lc}
\hline Parameter & Value \\
\hline$m$ & $56 \mathrm{~kg}$ \\
$J_{x}$ & $0.6366 \mathrm{~kg} \cdot \mathrm{m}^{2}$ \\
$J_{y}$ & $0.6366 \mathrm{~kg} \cdot \mathrm{m}^{2}$ \\
$J_{z}$ & $0.7958 \mathrm{~kg} \cdot \mathrm{m}^{2}$ \\
$k_{i 0}$ & $1140 \mathrm{~N} / \mathrm{A}$ \\
$x_{0}$ & $250 \mu \mathrm{m}$ \\
$L$ & $60.5 \mathrm{mH}$ \\
$R$ & $2.5 \Omega$ \\
$R_{r}$ & $12000 \mathrm{r} / \mathrm{min}$ \\
$l_{s}$ & $0.177 \mathrm{~m}$ \\
$l_{m}$ & $0.113 \mathrm{~m}$ \\
$k_{h 0}$ & $2.83 \mathrm{~N} / \mu \mathrm{m}$ \\
\hline
\end{tabular}

4.1. Simulation and Experimental Setup. The large magnetically suspended control moment gyroscope (MSCMG), as shown in Figure 3, is employed as the simulation and experimental setup since its MSFW has strong gyroscopic effects.

The well-known decentralized PD plus filter cross feedback controller is employed. The main parameters of the MSCMG and the controllers used in simulation and experiments have been listed in Tables 1 and 2. In Table $1, x_{0}$ is the air gap of the radial magnetic bearings; $k_{i 0}$ and $k_{h 0}$ are the current stiffness and displacement stiffness of the radial magnetic bearings under the conditions $i_{\gamma}=0$ and $h_{m \gamma}=$ $0(\gamma=a x, a y, b x, b y)$; and $R$ and $L$ are the coil resistance and inductance of the radial magnetic bearings. In Table 2, $k_{a m}$ and $i_{c}$ are the proportional and feedback coefficients of the current controller for the MSFW system, which are the same as those of the proposed controller; $k_{p}$ and $k_{d}$ are the proportional, integral, and differential coefficients of the PD plus cross feedback controller for the MSFW system; $k_{h c}$ and $k_{l c}$ are the cross coefficients of the high-pass and low-pass filters, respectively; $k_{c}$ is the total cross coefficient; and $f_{h}$ and $f_{l}$ are the cut-off frequencies of the two-order high-pass and low-pass filters.

Considering the bandwidths of interest of the current loop and the position loop of the MSFW and the simplicity of the realization, second-order antialias filters are introduced 
TABLE 2: Parameters of the controller.

\begin{tabular}{lc}
\hline Parameter & Value \\
\hline$k_{a m}$ & 2.0 \\
$k_{p}$ & 2.5 \\
$k_{h c}$ & 0.0045 \\
$k_{c}$ & 1.0 \\
$f_{l}$ & $30 \mathrm{~Hz}$ \\
$k_{r i}$ & 0.04 \\
$k_{c p}$ & 30 \\
$i_{c}$ & 10 \\
$k_{d}$ & 0.008 \\
$k_{l c}$ & 0.00015 \\
$f_{h}$ & $300 \mathrm{~Hz}$ \\
$k_{r p}$ & 0.8 \\
$k_{r d}$ & 0.3 \\
\hline
\end{tabular}

into both the displacement and current $\mathrm{AD}$ sampling of the MSFW system, whose transfer function is given as

$$
g_{f}(s)=\frac{1}{\left(3.3 \times 10^{-5} s+1\right)^{2}} .
$$

As far as the realization and implementation in hardware are concerned, a TMS320C32 digital signal processor (DSP) is employed. Both the sampling time and servo time are set to $150 \mu \mathrm{s}$.

4.2. Simulation Results. Figure 4 shows a plot of the nondimensional damping versus nondimensional stiffness for different growth rate. Gyroscopic effects result in either nutation (forward whirl) or precession (backward whirl). It also shows the growth rate, $\alpha$ (real part of the eigenvalue), and the frequency of oscillation, $\beta$ (imaginary part of the eigenvalue). The nondimensional stiffness is given by (16) and the nondimensional damping is given by (15). If the real part of the eigenvalue $\alpha$ is defined as positive, the vibrations grow in time and the system is unstable. If $\alpha$ is defined as negative, the vibrations decay in time and the system is stable. The condition that $\alpha$ equals zero defines the stability boundary. The imaginary part of the eigenvalue, $\beta$, is a parameter which varies from 0 to $\pi / 2$. The nondimensional speed of the flywheel is 0.1 for nutation, 0 for zero rotor speed, and -0.1 for precession. Nondimensional negative stiffness of 0.2 is assumed. From Figure 4, it can be drawn that a stable region is similar to that of the single-DOF magnetic bearing except it is now limited by nutation.

Figure 5 shows the dynamic stability map for a MSFW with time delay for various values of rotor speed, $\Omega$. Note that the stiffness must be greater than the negative stiffness for the system to be stable. The area from the trajectory to the left line $\left(\tau_{0}^{2}=0.2\right)$ represents the stability limits resulting from the forward whirl (the real part of the eigenvalue, $\sigma$, equals zero). The stable region becomes smaller for higher nondimensional rotor speeds. As for the traditional decentralized PD control, there is no stable region for nondimensional rotor speeds higher than 0.9. Contrarily, as for the decentralized

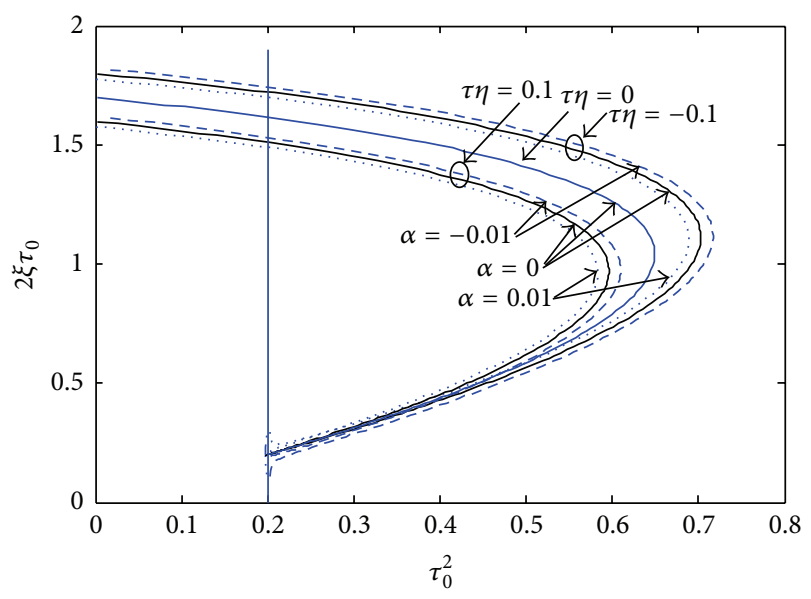

FIGURE 4: Stable region for the MSFW using a centralized controller with time delay.

PD plus cross feedback control, the nondimensional rotor speeds can reach about 1.7, and there is larger stable region than that of the case without cross feedback control at every rotor speed. This demonstrates that the cross feedback control algorithm can effectively improve the system stability, which is in accordance with the analysis made above.

To further prove the correctness of Theorem 1, that is, the phase compensation method, the positive and negative frequency characteristics analysis method [33] is employed. At $\Omega=40 \mathrm{~Hz}$, three different phase compensation methods have been compared, that is, the decentralized channels compensation, the cross-channel compensation, and the combinational compensation. To be fair, the three methods employ the same compensation filters:

$$
g_{c}(s)=\frac{2.2 s^{2}+3080 s+1.1 \times 10^{5}}{0.2 s^{2}+2000 s+1.13 \times 10^{5}} .
$$

The control channel transfer function of the MSFW system without phase compensation can be described as

$$
G_{0}(s)=g_{a}(s) g_{f}(s)\left[g_{b}(s)+j g_{\mathrm{cr}}(s)\right] e^{-\tau s} .
$$

According to the positive and negative frequency characteristics stability criterion [33], the control channel transfer function with the decentralized channel can be described as

$$
G_{1}(s)=g_{a}(s) g_{f}(s)\left[g_{b}(s) g_{c}(s)+j g_{\mathrm{cr}}(s)\right] e^{-\tau s} .
$$

Similarly, the control channel transfer function with the cross-channel compensation can be described as

$$
G_{2}(s)=g_{a}(s) g_{f}(s)\left[g_{b}(s)+j g_{c r}(s) g_{c}(s)\right] e^{-\tau s} .
$$

And the control channel transfer function with the combinational phase compensation can be described as

$$
G_{2}(s)=g_{a}(s) g_{f}(s)\left[g_{b}(s)+j g_{\mathrm{cr}}(s)\right] g_{c}(s) e^{-\tau s} .
$$

Figure 5 shows the comparative simulation results among different compensation methods, where "without compensation" demonstrates that the control system does not introduce 


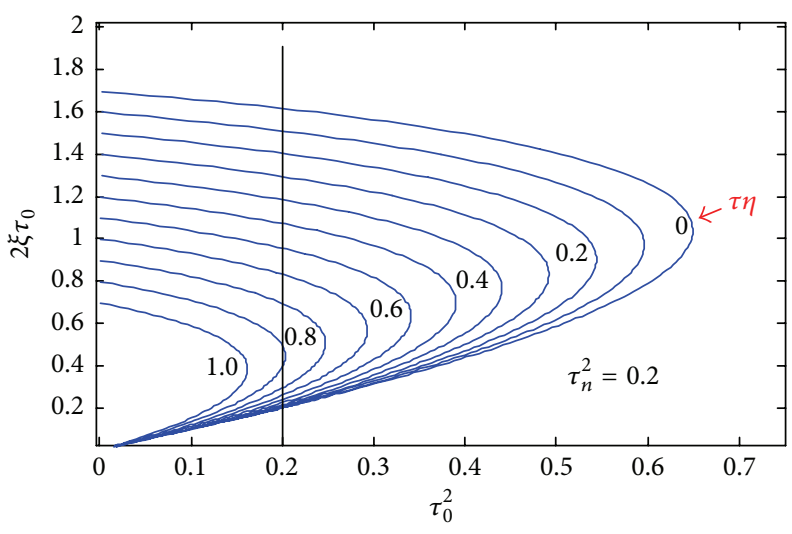

(a)

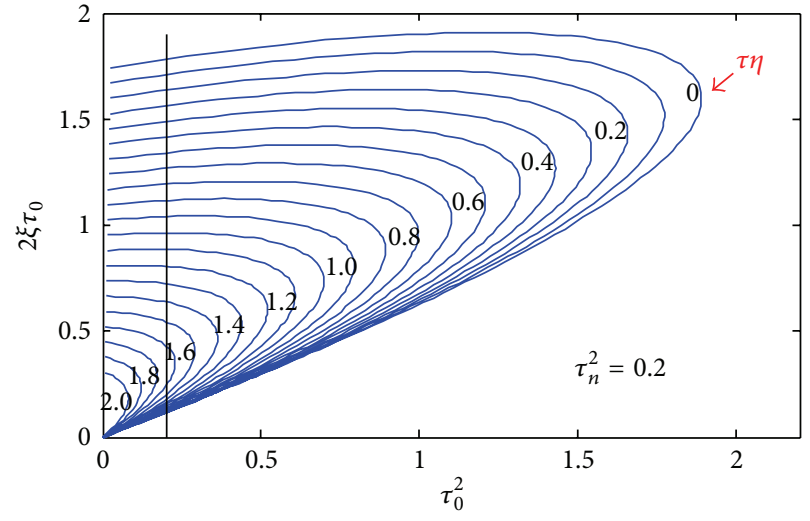

(b)

FIGURE 5: Dynamic stability map for a MSFW with time delay for various values of speed with different controller. (a) Decentralized PD controller. (b) Decentralized PD control plus cross feedback control.

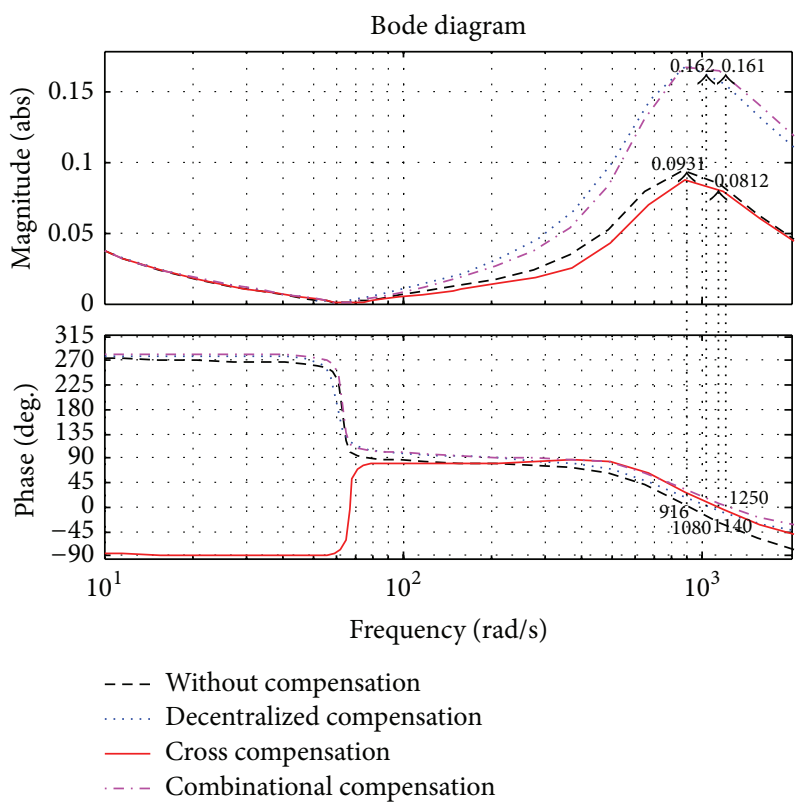

FIGURE 6: Comparative positive frequency characteristics curves among different compensation methods.

any phase compensation. According to the nutation and precession stability criterion of the magnetically suspended with strong gyroscopic effects [33], at rotor speed $\Omega$, the rotor speed stability margin $\Omega_{r}$ can be described as

$$
\Omega_{r}=\frac{J_{r} \omega_{n}}{J_{z}}+\frac{2 k_{h} l_{m}^{2}-2 l_{m} l_{s} k_{i} k_{s} i_{n}}{J_{z} \omega_{n}}-\Omega,
$$

where $i_{n}$ is the gain of the control channel at the frequency $\omega_{n}$.

From Figure 6, the critical whirling frequencies and their corresponding gain can be obtained: $\omega_{n 0}=916 \mathrm{rad} / \mathrm{s}, \omega_{n 1}=$ $1080 \mathrm{rad} / \mathrm{s}, \omega_{n 2}=1140 \mathrm{rad} / \mathrm{s}, \omega_{n 3}=1250 \mathrm{rad} / \mathrm{s}, i_{n 0}=0.0931$, $i_{n 1}=0.162, i_{2}=0.0812$, and $i_{3}=0.161$. According to the nutation stability criterion [33], it can be resolved that $\Omega_{r 0}=$
$22.0 \mathrm{~Hz}, \Omega_{r 1}=7.0 \mathrm{~Hz}, \Omega_{r 2}=68.5 \mathrm{~Hz}$, and $\Omega_{r 3}=41.6 \mathrm{~Hz}$. It is obvious that, among the three phase compensation methods, the cross-channel phase compensation improves the system nutation stability most greatly. It is necessary to note that the traditional combinational compensation method does have some effect in improving the system nutation stability. However, it is not the most effective way compared with the cross-channel compensation. Additionally, the decentralized channel compensation method endangers the system nutation stability margin instead of improving it. All in all, among the three compensation methods, the crosschannel compensation is the most effective way, which is in accordance with the analysis made above.

4.3. Experimental Results. To further prove the effectiveness and superiority of the phase compensation method, a comparison of experiments between the cross-channel compensation and the decentralized channels compensation has been performed. At $\Omega=40 \mathrm{~Hz}$, these two different compensation methods are performed. Figures 7 and 8 show the relationship between the nutation amplitude and the rotor speed with the two different compensation methods, respectively.

As shown in Figure 7, the nutation amplitude increases heavily with the rotor speed with the decentralized compensation method. And it increases to about $-49 \mathrm{~dB}$ at $45 \mathrm{~Hz}$, which demonstrates that the system will lose its nutation stability if the rotor speed continues to increase. On the contrary, the nutation amplitude keeps at about $-54 \mathrm{~dB}$ in the rotor speed of 40 to $70 \mathrm{~Hz}$ with the cross-channel compensation method as shown in Figure 8. This shows that the system nutation stability has been improved greatly by the cross-channel compensation instead of the decentralized channel compensation.

\section{Conclusion}

This paper studied the effects of time delay on the stability of the rotation modes and presented an effective phase 


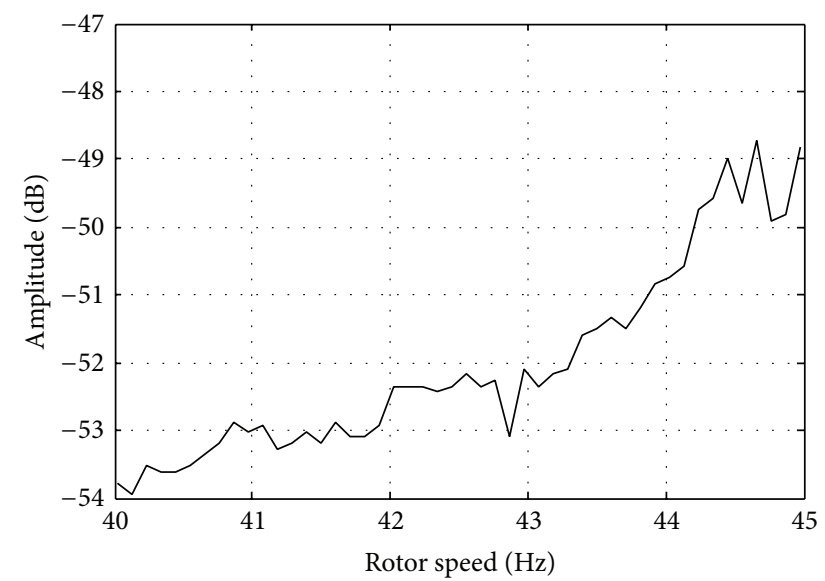

FIGURE 7: Nutation amplitude with rotor speed by the decentralized channel compensation.

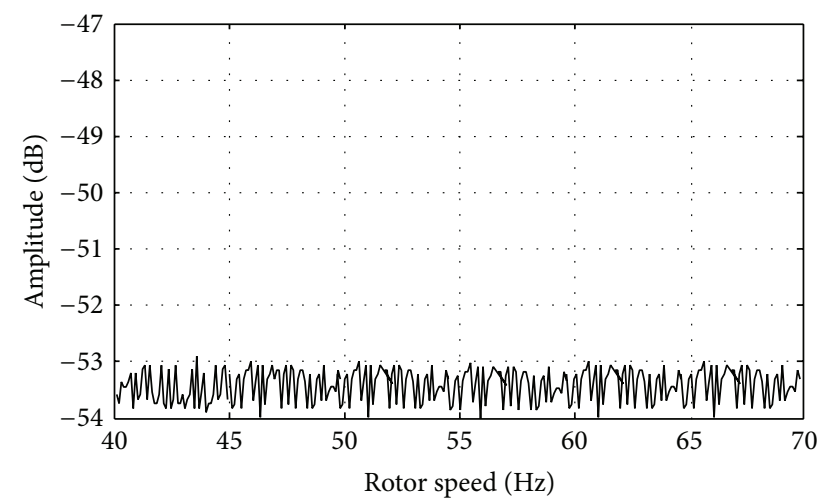

FIGURE 8: Nutation amplitude with temperature by the crosschannel compensation.

compensation method based on the cross-channels for the MSFW with strong gyroscopic effects. Analysis, simulation, and experimental results yield the following conclusion:

(1) The larger the rotor speed is, the smaller the stability region is.

(2) The forward whirl limited the region of stability.

(3) As for high speed MSFW, cross feedback control can effectively reject the nutation and precession instability.

(4) The delay of the cross-channel is the main factor influencing the nutation stability of the high-speed MSFW system, and the cross-channel compensation is the most effective way to improve the nutation stability.

To sum up, the presented local stability analysis method can effectively demonstrate the system stability region of the MSFW with strong gyroscopic effects and time delay, and the presented phase compensation method can greatly improve the system nutation stability. The global stability and semistable oscillations are the next topics in the near future.

\section{Competing Interests}

The authors declare that there is no conflict of interests regarding the publication of this paper.

\section{Acknowledgments}

This work was supported by the National Natural Science Foundation of China under Grants 51475472 and 61403396.

\section{References}

[1] J. Tang, J. Sun, J. Fang, and G. Shuzhi Sam, "Low eddy loss axial hybrid magnetic bearing with gimballing control ability for momentum flywheel," Journal of Magnetism and Magnetic Materials, vol. 329, pp. 153-164, 2013.

[2] S.-Y. Chen and F.-J. Lin, "Decentralized PID neural network control for five degree-of-freedom active magneticbearing," Engineering Applications of Artificial Intelligence, vol. 26, no. 3, pp. 962-973, 2013.

[3] C. Zhang and K. J. Tseng, "Design and control of a novel flywheel energy storage system assisted by hybrid mechanicalmagnetic bearings," Mechatronics, vol. 23, no. 3, pp. 297-309, 2013.

[4] Z. Yu, G. Zhang, Q. Qiu, L. Hu, B. Zhuang, and M. Qiu, "Analyses and tests of HTS bearing for flywheel energy system," IEEE Transactions on Applied Superconductivity, vol. 24, no. 3, Article ID 5700405, 2014.

[5] H. Bangcheng, Z. Shiqiang, W. Xi, and Q. Yuan, "Integral design and analysis of passive magnetic bearing and active radial magnetic bearing for agile satellite application," IEEE Transactions on Magnetics, vol. 48, no. 6, pp. 1959-1966, 2012.

[6] J. Fang, S. Zheng, and B. Han, "AMB vibration control for structural resonance of double-gimbal control moment gyro with high-speed magnetically suspended rotor," IEEE/ASME Transactions on Mechatronics, vol. 18, no. 1, pp. 32-43, 2013.

[7] Y. Ren and J. Fang, "High-precision and strong-robustness control for an MSCMG based on modal separation and rotation motion decoupling strategy," IEEE Transactions on Industrial Electronics, vol. 61, no. 3, pp. 1539-1551, 2014.

[8] Y. Ren and J. Fang, "Modified cross feedback control for a magnetically suspended flywheel rotor with significant gyroscopic effects," Mathematical Problems in Engineering, vol. 2014, Article ID 325913, 11 pages, 2014.

[9] X. Zhang, M. Liu, B. Wang, H. Chen, and Z. Wang, "A wide measurement range and fast update rate integrated interface for capacitive sensors array," IEEE Transactions on Circuits and Systems I: Regular Papers, vol. 61, no. 1, pp. 2-11, 2014.

[10] H. Chen, J. Gao, S. Su, X. Zhang, and Z. Wang, "A visual-aided wireless monitoring system design for total hip replacement surgery," IEEE Transactions on Biomedical Circuits and Systems, vol. 9, no. 2, pp. 227-236, 2015.

[11] Y. Ren and J. Fang, "Current-sensing resistor design to include current derivative in PWM H-bridge unipolar switching power amplifiers for magnetic bearings," IEEE Transactions on Industrial Electronics, vol. 59, no. 12, pp. 4590-4600, 2012.

[12] J. Fang and Y. Ren, "Self-adaptive phase-lead compensation based on unsymmetrical current sampling resistance network for magnetic bearing switching power amplifiers," IEEE Transactions on Industrial Electronics, vol. 59, no. 2, pp. 1218-1227, 2012. 
[13] J. C. Ji, "Dynamics of a Jeffcott rotor-magnetic bearing system with time delays," International Journal of Non-Linear Mechanics, vol. 38, no. 9, pp. 1387-1401, 2003.

[14] J. C. Ji and A. Y. T. Leung, "Non-linear oscillations of a rotormagnetic bearing system under superharmonic resonance conditions," International Journal of Non-Linear Mechanics, vol. 38, no. 6, pp. 829-835, 2003.

[15] H. Wang and J. Liu, "Stability and bifurcation analysis in a magnetic bearing system with time delays," Chaos, Solitons and Fractals, vol. 26, no. 3, pp. 813-825, 2005.

[16] Z. Li, J. B. Park, Y. H. Joo, B. Zhang, and G. Chen, "Bifurcations and chaos in a permanent-magnet synchronous motor," IEEE Transactions on Circuits and Systems-I: Fundamental Theory and Applications, vol. 49, no. 3, pp. 383-387, 2002.

[17] J. C. Ji, "Stability and Hopf bifurcation of a magnetic bearing system with time delays," Journal of Sound and Vibration, vol. 259, no. 4, pp. 845-856, 2003.

[18] A. F. Kascak, G. V. Brown, R. H. Jansen, and T. P. Dever, "Stability limits of a PD controller for a flywheel supported on rigid rotor and magnetic bearings," in Proceedings of the AIAA Guidance, Navigation, and Control Conference and Exhibit, pp. 1144-1155, San Francisco, Calif, USA, August 2005.

[19] A. F. Kascak, G. V. Jansen, R. H. Jansen et al., "Stability limits of a PD controller for a flywheel supported on rigid rotor and magnetic bearings," in Proceedings of the AIAA Guidance, Navigation, and Control Conference and Exhibit, San Francisco, Calif, USA, August 2005.

[20] H. S. Bauomy, "Stability analysis of a rotor-AMB system with time varying stiffness," Journal of the Franklin Institute, vol. 349, no. 5, pp. 1871-1890, 2012.

[21] J. I. Inayat-Hussain, "Nonlinear dynamics of a magnetically supported rigid rotor in auxiliary bearings," Mechanism and Machine Theory, vol. 45, no. 11, pp. 1651-1667, 2010.

[22] S. B. Andersen, S. Enemark, and I. F. Santos, "Dynamics and stability of rigid rotors levitated by passive cylinder-magnet bearings and driven/supported axially by pointwise contact clutch," Journal of Sound and Vibration, vol. 332, no. 25, pp. 6637-6658, 2013.

[23] T. Sugai, T. Inoue, and Y. Ishida, "Nonlinear theoretical analysis of contacting forward whirling vibration of a rotating shaft supported by a repulsive magnetic bearing," Journal of Sound and Vibration, vol. 332, no. 11, pp. 2735-2749, 2013.

[24] Y.-C. Pei, "Stability boundaries of a spinning rotor with parametrically excited gyroscopic system," European Journal of Mechanics-A/Solids, vol. 28, no. 4, pp. 891-896, 2009.

[25] C.-C. Fan and M.-C. Pan, "Experimental study on the whip elimination of rotor-bearing systems with electromagnetic exciters," Mechanism and Machine Theory, vol. 46, no. 3, pp. 290-304, 2011.

[26] Y. Okada, B. Nagai, and T. Shimane, "Cross-feedback stabilization of the digitally controlled magnetic bearing," Journal of Vibration, Acoustics, Stress, and Reliability in Design, vol. 114, no. 1, pp. 54-59, 1992.

[27] M. Ahrens, L. Kučera, and R. Larsonneur, "Performance of a magnetically suspended flywheel energy storage device," IEEE Transactions on Control Systems Technology, vol. 4, no. 5, pp. 494-502, 1996.

[28] T. P. Dever, G. V. Brown, K. P. Duffy, and R. H. Jansen, "Modeling and development of a magnetic bearing controller for a high speed flywheel system," in Proceedings of the 2nd International Energy Conversion Engineering Conference, AIAA 2004-5626, Providence, RI, USA, August 2004.
[29] G. V. Brown, A. Kascak, R. H. Jansen, T. P. Dever, and K. P. Duffy, "Stabilizing gyroscopic modes in magnetic-bearing-supported flywheels by using cross-axis proportional gains," in Proceedings of the AIAA Guidance, Navigation, and Control Conference and Exhibit, AIAA 2005-5955, San Francisco, Calif, USA, August 2005.

[30] M. Chen and C. R. Knospe, "Feedback linearization of active magnetic bearings: current-mode implementation," IEEE/ASME Transactions on Mechatronics, vol. 10, no. 6, pp. 632-639, 2005.

[31] S.-L. Chen and C.-C. Weng, "Robust control of a voltagecontrolled three-pole active magnetic bearing system," IEEE/ASME Transactions on Mechatronics, vol. 15, no. 3, pp. 381-388, 2010.

[32] K. Y. Zhu, Y. Xiao, and A. U. Rajendra, "Optimal control of the magnetic bearings for a flywheel energy storage system," Mechatronics, vol. 19, no. 8, pp. 1221-1235, 2009.

[33] J. Fang and Y. Ren, "Decoupling control of magnetically suspended rotor system in control moment gyros based on an inverse system method," IEEE/ASME Transactions on Mechatronics, vol. 17, no. 6, pp. 1133-1144, 2012.

[34] J. Fang and Y. Ren, "High-precision control for a single-gimbal magnetically suspended control moment gyro based on inverse system method," IEEE Transactions on Industrial Electronics, vol. 58, no. 9, pp. 4331-4342, 2011.

[35] J. Fang, Y. Ren, and Y. Fan, "Nutation and precession stability criterion of magnetically suspended rigid rotors with gyroscopic effects based on positive and negative frequency characteristics," IEEE Transactions on Industrial Electronics, vol. 61, no. 4, pp. 2003-2014, 2014.

[36] G. Bianchi, N. V. Kuznetsov, G. A. Leonov et al., "Limitations of PLL simulation: hidden oscillations in MatLab and SPICE," in Proceedings of the IEEE 7th International Congress on Ultra Modern Telecommunications and Control Systems, Brno, Czech Republic, October 2015. 


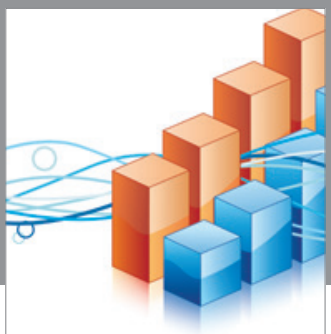

Advances in

Operations Research

vatem alat4

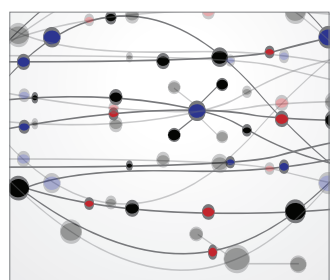

\section{The Scientific} World Journal
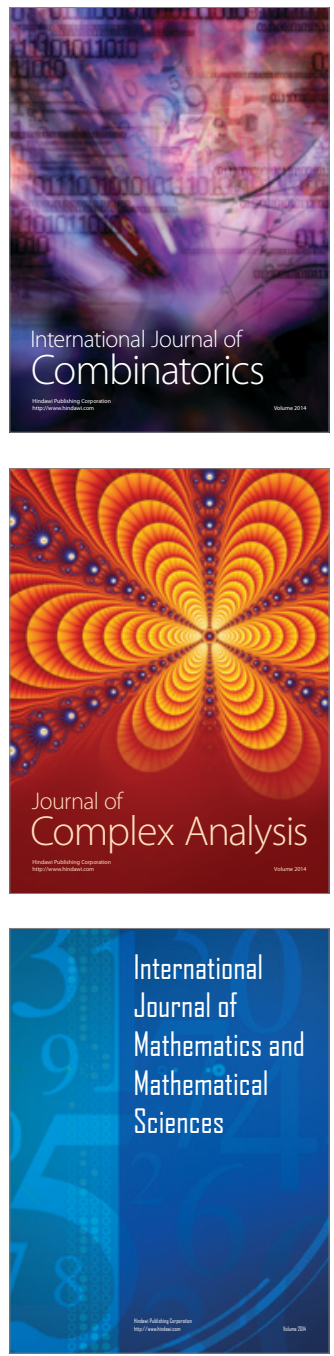
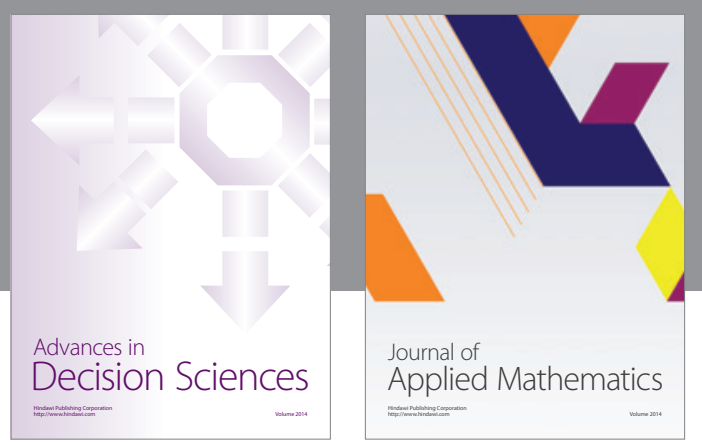

Algebra

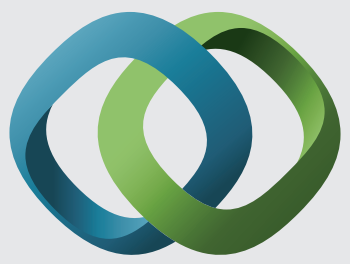

\section{Hindawi}

Submit your manuscripts at

http://www.hindawi.com
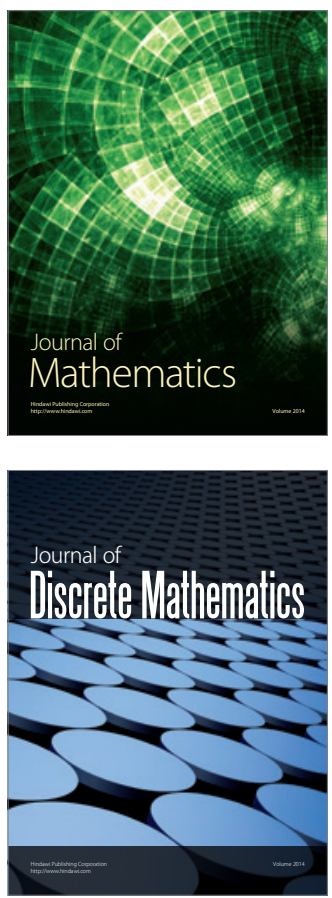

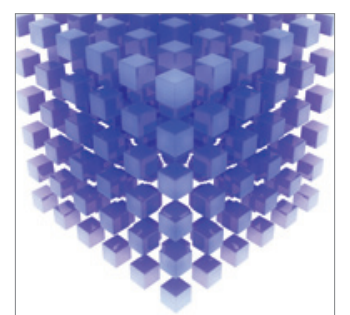

Mathematical Problems in Engineering
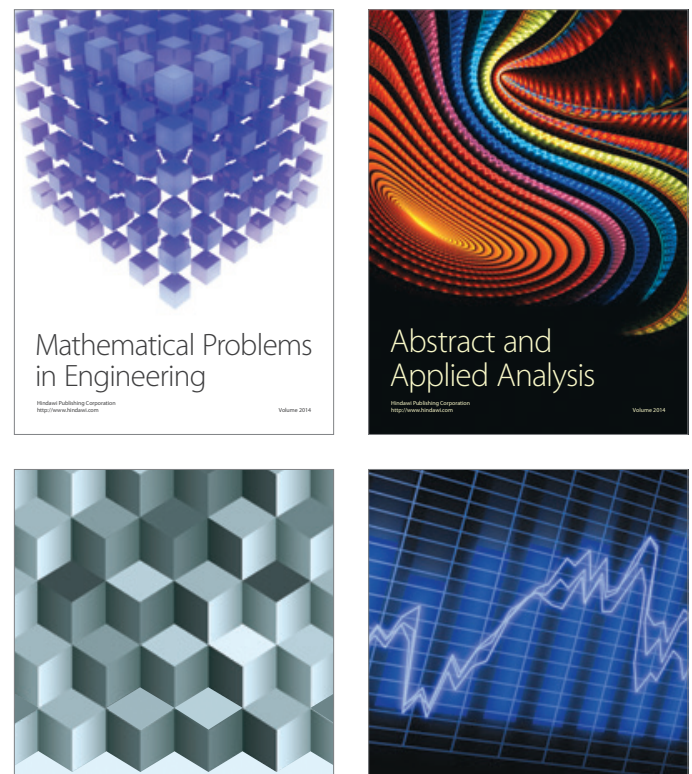

Journal of

Function Spaces

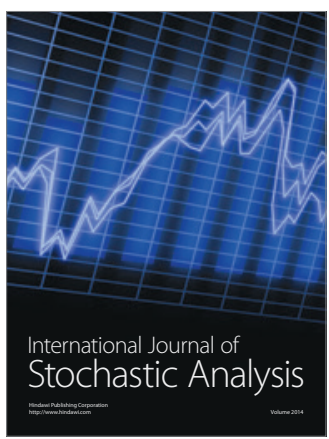

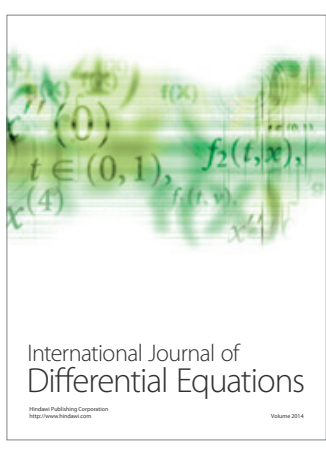
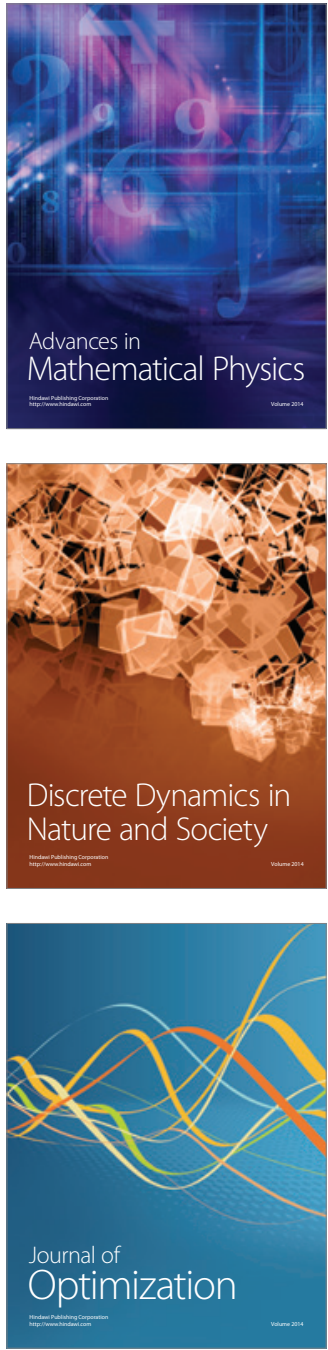